\title{
1 On the interaction between human IQGAP1 and actin
}

2

3 Damian J. Magill ${ }^{1}$, Elaine Hamilton ${ }^{1,3}$, Sally L. Shirran ${ }^{2}$, Catherine H. Botting ${ }^{2}$ and

4 David J. Timson ${ }^{1,4} *$

5

$6{ }^{1}$ School of Biological Sciences, Queen's University Belfast, Medical Biology Centre, 97

7 Lisburn Road, Belfast BT9 7BL. UK.

$8 \quad{ }^{2}$ School of Chemistry, Biomedical Sciences Research Complex, BMS Annexe, University of

9 St. Andrews, St. Andrews, KY16 9ST. UK.

$10{ }^{3}$ Current address: Belfast Metropolitan College, Castlereagh Campus, Montgomery Road,

11 Belfast, BT6 9JD. UK.

$12{ }^{4}$ School of Pharmacy and Biomolecular Sciences, University of Brighton, Huxley Building,

13 Lewes Road, Brighton BN2 4GJ. UK.

$14 *$ Author to whom correspondence should be addressed at: School of Pharmacy and

15 Biomolecular Sciences, University of Brighton, Huxley Building, Lewes Road, Brighton

16 BN2 4GJ. UK.

17 Telephone $\quad+44(0) 1273642090$

$18 \quad$ Fax $\quad+44(0) 1273642090$

19 Email d.timson@brighton.ac.uk 


\section{Abstract}

22 IQGAPs are eukaryotic proteins which integrate signals from various sources and pass these

23 on the cytoskeleton. Understanding how they do this requires information on the interfaces

24 between the proteins. Here, it is shown that the calponin homology domain of human

25 IQGAP1 (CHD1) can be crosslinked with $\alpha$-actin. The stoichiometry of the interaction was

26 1:1. A molecular model was built of the complex and associated bioinformatics analyses predicted that the interaction is likely to involve an electrostatic interaction between Lys-240 of $\alpha$-actin and Glu-30 of CHD1. These residues are predicted to be accessible and are not involved in many intra-protein interactions; they are thus available for interaction with binding partners. They are both located in regions of the proteins which are predicted to be

31 flexible and disordered; interactions between signalling molecules often involve flexible, disordered regions. The predicted binding region in CHD1 is well conserved in many eukaryotic IQGAP-like proteins. In some cases (e.g Dictyostelium discoideum and

Saccharomyces cerevisiae) protein sequence conservation is weak, but molecular modelling reveals that a region of charged, polar residues in a flexible $\mathrm{N}$-terminus is structurally well conserved. Therefore we conclude that the calponin homology domains of IQGAP1-like proteins interact initially through the electrostatic interaction identified here and that there may be subsequent conformational changes to form the final complex.

40 Key words: $\alpha$-actin; protein flexibility; protein-protein crosslinking; calponin homology 41 domain; CHD; IQGAP-like protein 


\section{Introduction}

43 The IQGAPs are a family of cytoskeletal scaffolding proteins in fungi and animals [1-10].

44 The name IQGAP is derived from two of the domains present in this group of proteins: the IQ, and the Ras GTPase-activating (GAP) related domains. They link a diverse range of signalling pathways, including calcium-calmodulin, small GTPases, kinases and growth factor receptors to the actin cytoskeleton [11-24]. In addition, they also help control the microtubule system indirectly through CLIP-170 and APC $[25,26]$. Not surprisingly, since they sit at the interface of many cellular processes, they have been implicated in diseases including cancer, heart disease and infection by bacteria and viruses [27-33]. In the last decade, considerable advances have been made in the understanding of the IQGAP family's cellular roles. This is especially true of the best characterised member of the family, human IQGAP1. However, many molecular-level details remain to be discovered. Although high resolution structures are available for some domains, there is no structure of a complete IQGAP; therefore there is little information about how the various domains in the protein communicate [34-37]. There is also only limited data available on how the protein interacts with its many binding partners. Such information would illuminate cellular level studies and may be of value in the event that it is shown to be desirable to disrupt these interactions for the development of novel anti-cancer or anti-infective drugs.

60

61 It is known that interaction with actin occurs through the calponin homology domain (CHD),

62 located at the $\mathrm{N}$-terminus of the protein $[13,38,39]$. The functional consequence of this

63 interaction is the bundling of actin filaments [40-42]. The CHD also provides an interaction

64 site for calmodulin, although this protein interacts primarily through IQ-motifs located towards the middle of the primary sequence $[13,43,44]$. Here a crosslinking protocol was used to confirm the actin/CHD interaction and determine its stoichiometry. Molecular 
modelling of the complex between the two molecules was undertaken to predict the site of interaction.

\section{Materials and Methods}

Proteins

72

A protein containing residues 2-215 from human IQGAP1 (which encompass the CHD and is referred to as CHD1 hereafter) was expressed in, and purified from, Escherichia coli as described previously [44]. Rabbit skeletal muscle actin was purchased from Cytoskeleton Inc. (Denver, CO, USA) as freeze dried powder. The powder was reconstituted in G-actin buffer (5 mM Tris-HCl, pH 8.0, $0.2 \mathrm{mM} \mathrm{CaCl}_{2}, 0.2 \mathrm{mM}$ ATP, $0.5 \mathrm{mM}$ DTT) to a final concentration of $1 \mathrm{mg} \mathrm{ml}^{-1}$, placed on ice for $60 \mathrm{~min}$ and then centrifuged at $13,000 \mathrm{~g}$ at $4{ }^{\circ} \mathrm{C}$ for $15 \mathrm{~min}$. The supernatant was removed and assumed to be G-actin. Protein concentrations were estimated by the method of Bradford [45], using BSA as a standard.

\section{Crosslinking}

Actin $(23 \mu \mathrm{M})$ and CHD1 $(27 \mu \mathrm{M})$ were mixed and incubated at $37^{\circ} \mathrm{C}$ for $60 \mathrm{~min}$. After this time, 1-ethyl-3-(3-dimethylaminopropyl)carbodiimide (EDC) was added to a final concentration of $70 \mathrm{mM}$ and the reaction allowed to proceed for $60 \mathrm{~min}$. The reaction was stopped by the addition of SDS-PAGE loading buffer (120 mM TrisHCl $\mathrm{pH} 6.8,4 \%$ (w/v) SDS, $20 \%(\mathrm{v} / \mathrm{v})$ glycerol, $5 \%(\mathrm{w} / \mathrm{v})$ bromophenol blue, $1 \%(\mathrm{w} / \mathrm{v}) \mathrm{DTT})$ and heating at $95{ }^{\circ} \mathrm{C}$ for 5 min. Results were analysed by SDS-PAGE.

\section{Mass spectrometry}

The gel band was excised and cut into $1 \mathrm{~mm}$ cubes. These were then subjected to in-gel digestion, using a ProGest Investigator in-gel digestion robot, using standard protocols. 
92 Briefly the gel cubes were destained by washing with acetonitrile and subjected to reduction and alkylation before digestion with trypsin at $37^{\circ} \mathrm{C}$. The peptides were extracted with $10 \%$

94 formic acid and concentrated down to $20 \mu \mathrm{L}$ (SpeedVac, ThemoSavant). They were then separated using an UltiMate nanoLC (LC Packings, Amsterdam) equipped with a PepMap

96 C18 trap \& column, using a 60 min gradient of increasing acetonitrile concentration, containing $0.1 \%$ formic acid (5-35\% acetonitrile in $35 \mathrm{~min}$ respectively, 35-50\% in a further 20 min, followed by $95 \%$ acetonitrile to clean the column). The eluant was sprayed into a QStar XL tandem mass spectrometer (Applied Biosystems, Foster City, CA) and analysed in

100 Information Dependent Acquisition (IDA) mode, performing $1 \mathrm{sec}$ of MS followed by $3 \mathrm{sec}$ 101 MSMS analyses of the 2 most intense peaks seen by MS. These masses are then excluded from analysis for the next $60 \mathrm{sec}$. MS/MS data for doubly and triply charged precursor ions was converted to centroid data, without smoothing, using the Analyst QS1.1 mascot.dll data import filter with default settings. The MS/MS data file generated was analysed using the Mascot 2.1 search engine (Matrix Science, London, UK) against the UniRef 100 database, Jan 2008 (5247086 sequences) with no species restriction. The data was searched with tolerances of 0.2 Da for the precursor and fragment ions, trypsin as the cleavage enzyme, one missed cleavage, carbamidomethyl modification of cysteines as a fixed modification and methionine oxidation selected as a variable modification. Molecular modelling of the actin and IQGAPI CHD interface

112 Models of human IQGAP1 residues 2 - 215 and rabbit skeletal muscle $\alpha$-actin (which is

$113100 \%$ identical to the human $\alpha$-actin sequence; UniProt accession numbers: human P46940,

114 rabbit P68135) were constructed using the Phyre2 server in intensive mode [46] and

115 displayed using PyMol (www.pymol.org). These were then minimised using the YASARA

116 force field [47]. Patchdock was utilised in the docking of ADP with actin and Firedock for 
117 refining of these results $[48,49]$. The first, most energetically favourable result was taken and

118 minimised using YASARA. Protein flexibility was predicted using FRODAN to generate 500

119 conformers in the momentum mode [50]. The HADDOCK flexible docking server was used

120 to model potential interactions between actin and CHD1 [51]. The postulated binding regions

121 were used as targets and HADDOCK was set to define its own passive interacting residues.

122 Only the most energetically favourable structure was used. Models were subjected to the

123 structure validation tools of the WHATIF server to check for potential collisions and to

124 validate bond lengths, planarity and dihedral angles of residues [52]. The xWalk algorithm

125 was used to calculate Euclidean distances between the potential cross linked residues in the

126 HADDOCK structure, whilst the ESBRI and PIC solvent accessibility tools allowed

127 calculation of potential salt bridges and solvent accessibility of the residues respectively [53-

128 55]. Residue accessibility was calculated using GETAREA [56].

130 Multiple sequence alignment, structural alignments, residue conservation and in silico

131 prediction of probable interacting residues

132 Multiple sequence alignment of CHDs was carried out using Clustal Omega [57, 58].

133 Sequences were obtained from NCBI using the psiBLAST tool to obtain distant members of

134 the eukaryotic lineage [59]. Sequences that were true IQGAP1 homologues and IQGAP1-like

135 proteins were used as well as the other human and mouse isoforms. The alignment was used

136 along with the Scorecons server to calculate residue conservation at each position based upon

137 the Valdar01 score [60]. The EMBOSScons tool (http://www.ebi.ac.uk/emboss) allowed for a

138 consensus sequence of the region to be deduced. Models of the aligned sequences were built

139 in Phyre2 and minimised for use in structural alignments. These were carried out in PyMol

140 using the align function. The WHATIF server was used to calculate residue accessibility in

141 both actin and CHD1 [52]. The programs DisEMBL and DISOclust were employed to 
142 calculate intrinsically disordered domains in the models [61, 62]. The ProFace server was

143 used to analyse the HADDOCK model and to provide the most probable interface residues

144 [63]. NNcon is a program that was used to analyse and provide a readout of intraprotein

145 interactions. Therefore, it was used to identify residues which were not involved in

146 intraprotein contacts and which will, therefore, be more available for protein-protein

147 interactions [64].

Prediction of the biochemical effects of missense mutations on the IQGAP1 CHD

150 The WHATIF server was used to mutate the potential cross linked residues in the CHD1 to

151 alanines. The resulting model was subject to analysis using the MuPro, SNPeffect and Site

152 directed mutator programs in order to predict potential effects on the protein's functionality

153 [52, 65-67]. The SNPeffect server uses four different programs: TANGO looks at how a

154 given mutation affects the propensity of a protein to aggregate, WALTZ focuses on the

155 effects the mutation may have on the tendency of a protein to form amyloids, LIMBO looks

156 at changes in a proteins ability to bind chaperones and FOLDX analyses the overall effects of

157 the mutation on the proteins stability. The mutated CHD model was also docked to actin

158 using HADDOCK (as described above for the wild type protein) in order to predict changes

159 in predicted energies and root mean square deviations (rmsd).

160

161 Results

162 Actin and the CHD from IQGAPIcan be crosslinked

163 When actin and CHD1 were mixed in the presence of the crosslinker EDC, an additional

164 band was observed at $\sim 70 \mathrm{kDa}$ (figure 1). This band did not appear when the proteins were

165 mixed in the absence of crosslinker or when each protein alone was exposed to EDC.

166 Therefore, it is likely to correspond to the covalently linked actin-CHD1 complex. The 
167 estimated molecular mass of the complex is consistent with it containing one molecule of actin (42 kDa) and one of CHD1 (26 kDa including the hexahistidine tag).

170 Bands were excised from these gels and subjected to in-gel digestion with trypsin and the

171 resultant peptides analysed by mass spectrometry. Bands corresponding to CHD1 plus

172 crosslinker and actin plus crosslinker resulted in 22 and 26 peptides with Mascot Ion Scores

173 above the Identity threshold (95\% confidence) matching to each sequence respectively. In the

174 band corresponding to the postulated crosslinked complex, peptides from both actin (16

175 peptides) and CHD1 (7 peptides) could be identified. This confirmed that the additional band

176 did, indeed, correspond to an actin-CHD1 complex. Interestingly, only peptides from $\alpha$-actin

177 were isolated; therefore bioinformatics and modelling studies concentrated on this isoform.

CHD1 and actin models were docked to predicted interaction sites

180 Phyre 2 modelled both proteins with greater than $90 \%$ accuracy and these were virtually

181 indistinguishable from the experimental structures following minimisations and alignment.

182 Both models were validated successfully by the WHATIF server.

184 The HADDOCK server docked the structure and the first, most favourable result, with a Z-

185 Score of -2.2 was taken and minimised using YASARA. xWalk was then utilised to find the

186 closest point of contact and this was shown to be between Lys-240 of actin and Glu-30 of

187 CHD1 at a Euclidean distance of $5.2 \AA$. The HADDOCK predicted complex is shown in

188 Figure 2. The top complex highlights residue Lys 240 of actin and residues Glu 29, 30, 33,

189 and Asp 32 of CHD whilst the bottom complex presents the docked structure with a semi-

190 transparent overlay of the surface of the two proteins.. xWalk showed that the solvent

191 accessible surface distance is $4.5 \AA$; a similar result was calculated by ESBRI (4.45 $\AA$ ). The 
192 PIC solvent accessibility tool validated these residues as being capable of ionic interaction.

193 While this alone does not prove that these two residues are part of the interaction site it is

194 consistent with the favourable HADDOCK energies associated with the docking process

195 (Table 1). Thus the model shown in figure 2 is a feasible representation of part of the

196 interface between actin and CHD1.

197

198 Any interaction site must be accessible in order to take part in a given interaction either

199 directly or following a conformational change exposing the site. Given this, the relative

200 accessibility of residues can be a good indicator of whether, or not, they can take part in

201 initial interactions. The accessibility of the actin and CHD1 residues of interest are given in

202 Table 2 as side, back and total overall residue accessibility. Of particular interest are charged

203 residues in the potential binding sites which are potentially crosslinkable by EDC. Residues

204 Glu-239 and Lys-240 of actin showed good overall accessibility (27.7 and $37.2 \AA^{2}$ total

205 accessibility respectively) as did the residues Glu-29, Glu-30, Glu-33 and Asp-32 (41.7, 38.0,

20613.5 and $44.8 \AA^{2}$ total accessibility respectively) of CHD1. It therefore seems likely that all

207 these residues are available and are potentially involved in the initial interaction. The Proface

208 analyser provides a readout of residues involved in the interface of the docked structure. The

209 results from this prediction are shown in Supplementary Figure S1 and include residues 28,

$21030,31,32,33,34$ from CHD1 and residues 237, 239 and 240 from the actin. The NNcon

211 program showed that up to residue 180 in actin there was generally potential for $>10$

212 intraprotein contacts between each amino acid and others within the protein. In some cases

213 there was $>40$ predicted interactions. After residue 180 , the number of potential interactions

214 per residue fell with some not taking part in any. For the putative actin site, residues 237,

215238,239 and 240 were shown to take part in $0,4,4$ and 5 intraprotein interactions

216 respectively. For CHD1, a block of residues from 21-26 and 28-43 inclusive are predicted to 
not be involved in any intraprotein interactions. These are the only large blocks of this nature

218 in the whole of the CHD1. Thus, the predicted binding regions in both proteins are free to

219 take part in protein - protein interactions.

220

The putative binding sites are predicted to be highly flexible

222 The interaction sites between proteins often involve highly mobile, flexible regions. This is often the case in signalling proteins where high specificity but short-lived interactions are required [68-70]. When interpreting flexibility predictions it is important to look at the flexibility of localised regions as opposed to limiting the analysis to individual residues. FIRST/FRODAN predicted that CHD1 has three highly flexible regions, which are the termini and a zone localised around residues 92-110 (Figure 3a). However, the N-terminus shows extraordinary flexibility and this extends almost as far as residue 40 and peaks at an rmsd of $9.2 \AA$ for Arg-24 (Figure 3b). The results for actin are equally intriguing. It is clear that it is a highly dynamic molecule but two regions stand out, which are those around residue 50 and the larger region spanning residues $224-250$. The rmsd peaked at $7.8 \AA$ at

232 Ala-233. The regions correspond to the two lobes in actin with the one around residue 50 being the DNase I binding loop [71]. The predicted interaction regions from both proteins therefore lie within the predicted areas of greatest flexibility. It is possible that, given the high flexibility of the two regions, all residues interact to varying extents by transient hydrogen

236 bonds and other non-covalent interactions. This may therefore be the initial interaction that

237 takes place between CHD1 and actin before a conformational change exposes other actin 238 binding sites. 
241 Intrinsic disorder was first calculated using DisEMBL which carries out these predictions

242 based upon three definitions of disorder [61]. Both actin and CHD1 disorders were calculated

243 for the loops/coils, hot loops and remark-465 definitions (Supplementary Figure S2). For

244 CHD1 there is extensive disorder for the loops/coils definition, significantly less for the hot

245 loops and none for the remark-465 definition (Supplementary Figure S2a). The remark-465

246 definition is less relevant in this case as it is generally only compatible with actual X-ray data

247 [61]. The hot loops definition is the most significant as this is a refinement of the first

248 loops/coils data. For CHD1, the fragment LTAEEM is implicated as being disordered. For

249 actin the results are much more profound. Only $13 \%$ of actin is disordered by the hot loops

250 definition and this includes the region TAASSSSLEK.

251

252 The DISOclust results add weight to the above predictions (Figure 3c,d) because they also 253 implicated the postulated regions. DISOclust scores are only stated to be of significance if 254 they exceed the 50\% threshold mark [62]. The CHD1 plot looks not unlike the FRODAN

255 results and it is common for disordered regions to also be highly flexible due to the lack of 256 three-dimensional structure. The postulated CHD1 binding site clearly lies within the 257 disordered region. Apart from the ends, only one other region in actin exceeds the DISOclust 258 threshold, residues 227-244 (Figure 3c).

260 Taken together, these predictions show that both the actin and CHD1 sites form part of 261 intrinsically disordered domains that are surface exposed. This may also implicate these 262 regions in binding of other partners.

264 In silico mutagenesis of the CHD1 site is stabilising and preserves overall structure, but is 265 predicted to reduce interaction with actin 
266 The residues Glu 29, 30, 33 and Asp 32 of CHD1 were mutated in silico to alanines using the

267 WHATIF mutate a residue tool [52]. MuPro [65] was used to analyse the effect of each of the

268 above mutations individually by predicting the change in the conformational stability of the

$269 \operatorname{protein}(\Delta \Delta \mathrm{G}$ value). MuPro predicted that each mutation would individually cause an

270 increase in the stability of the protein. The original and WHATIF model were then submitted

271 to the SDM server [67] and this too predicted that the protein would increase in stability but

272 also that it would most likely to be non-functional as a result (data not shown).

273

274 The SNPeffect server showed that the mutations have no effect on aggregation tendency,

275 amyloid propensity or the ability of the protein to bind chaperones. The FOLDX component,

276 however, predicted that the protein would increase slightly in stability. The fact that these

277 mutations were predicted to cause an increase in stability was surprising since it is common

278 for amino acid changes to destabilise proteins. However, in this case, it is possible that the

279 mutations cause an increase in stability via an elimination of charge repulsion between the

280 glutamates and aspartate. This, combined with the shorter alanine side chain, may enable the

281 protein to adopt a slightly more compact and stable structure that does not cause malign

282 effects such as aggregation. The loss of these potential interacting residues, the stability

283 changes and likely decrease in flexibility were postulated to diminish interaction with actin.

285 The mutated structure was taken and docked with wild type actin using HADDOCK using the

286 same docking parameters as wild type. The results were intriguing (Table 1) as there was a

287 substantial drop in electrostatic and Van der Waals energies, with the mutant-actin complex

288 having less than half the electrostatic energy of the complex with wild type. It is clear that

289 docking of the mutant is an unfavourable process compared to the wild type. 
Sequence alignments, between analogous proteins from different species, often identify the

293 functional, structural and/or evolutionary relationships between them [72]. Actin is one of the

294 most conserved eukaryotic proteins and as such it would be difficult to deduce potential

295 binding sites from structural and sequence information alone. CHD1 is not as strongly

296 conserved and some inferences can be drawn from its alignment. Sequences were compared

297 for the human IQGAP isoforms and lower eukaryotes such as Trachipleistophora hominis

298 and Caenorhabditis elegans (Supplementary Figure S3). It is be immediately obvious that

299 there is reasonable conservation of the region, especially the aspartate and adjacent

300 glutamate, and also a block of arginine residues following the postulated interaction region.

301 These arginines may also be involved in the interaction. When the EMBOSScons sequence

302 tool was used, it generated VDDERLSAEEMDERRR as the consensus sequence. The

303 Scorecons server shows $0.413,0.627,0.685,0.612,0.684,0.921,1.000,0.912,0.611,1.000$

304 and 0.640 as conservation values for the residues LTAEEMDERRR. In many cases, when a

305 residue is not conserved in a sequence it is replaced by an amino acid that could carry out the

306 same function e.g. polar threonine for serine, aspartate instead of glutamate and glutamine or

307 lysine instead of arginine.

308

309 However, when the CHD from the budding yeast (Saccharomyces cerevisiae) IQGAP-like

310 protein, Iqg1p, is aligned the following sequence lines up with the postulated actin binding

311 region: KYTVDLSNYSKIELRYYEF. This does contain largely polar and charged residues

312 and could be involved in the interaction, but the lack of precise sequence alignment means

313 that there must be differences between this protein and those from higher eukaryotes.

314 However, given that sequence defines structure, structure defines function and that different

315 sequences may produce similar overall structures, the structural alignment of proteins is often 
316 a much better indicator of deducing functionally equivalent sites. Therefore, Phyre2 was used

317 to construct models of CHDs from a variety of species and, following minimisation, these

318 were aligned to the human structure using PyMol. The predicted structures of human CHD1

319 aligned with the equivalent regions from IQGAP-like proteins from C. elegans, Hydra

320 vulgaris, and S. cerevisiae are shown in Figure 4a,b,c. Although the $S$. cerevisiae sequence

321 aligns only poorly with the human one, it is clear that the structures do align and, interestingly, Asp-93 aligns with the postulated actin-binding domain in human CHD1 (Figure 4c). In all three cases the N-termini of the CHDs are the unstructured, intrinsically

324 disordered regions of the proteins; this was supported by DISOclust and DisEMBL

325 predictions (data not shown).

As a further test of this hypothesis, the IQGAP-like protein from the soil amoeba

Dictyostelium discoideum was investigated (gapA; Uniprot O00899) [73, 74]. The significant feature of this protein is that it does not have any regions that are recognised as CHDs.

330 Therefore, residues 1-216 were taken and a Phyre 2 model produced and subsequently aligned. The resulting model (Figure 4d) is clearly different from others, but alignment with human CHD1 showed that the N-terminus of $D$. discoideum gapA aligned reasonably well with the postulated binding site in the human protein; coincidently this is also a negatively charged region with an abundance of polar residues (Figure 4d). So, although $D$. discoideum has no recognised CHD, it shows some structural similarity and, therefore, may bind actin through a novel, functionally equivalent domain.

\section{Discussion}

It has been proposed that there are two actin binding domains used by some CHD-containing

340 proteins [75-78]. However, in IQGAP1, one CHD is sufficient for actin binding [39]. The 
341 experimental work presented here demonstrated that the stoichiometry of the interaction is

342 1:1. Here we propose that the regions identified in this work (and the polar, intrinsically

343 disordered analogous regions in other IQGAP-like proteins) form at least part of the initial

344 interface between the proteins. We believe this could be a lower affinity interface associated

345 with transient hydrogen bonds and electrostatic interactions that precedes conformational

346 change and tighter binding at other sites. We further note that calmodulin has been

347 previously predicted to interact with the same part of the CHD and speculate that direct

348 competition for the binding site may be a regulatory mechanism [44]. Further analyses are

349 necessary to fully characterise the interactions between these proteins.

Acknowledgements

352 DM thanks the School of Biological Sciences, Queen's University, Belfast for a summer 353 studentship and EH thanks the Department of Employment and Learning, Northern Ireland

354 for a postgraduate studentship. The work was funded in part by grants from the BBSRC

355 (BB/D000394/1 To DJT) and by the Wellcome Trust [grant number GR06281AIA] which

356 funded the purchase of the QStar XL mass spectrometer at the BBSRC Mass Spectrometry

357 and Proteomics Facility, University of St Andrews and funded SLS.

358

359 
361 1. Briggs, M.W.; Sacks, D.B. IQGAP proteins are integral components of cytoskeletal

362 regulation. EMBO Rep 2003, 4, 571-574.

363 2. Briggs, M.W.; Sacks, D.B. IQGAP1 as signal integrator: $\mathrm{Ca}^{2+}$, calmodulin, $\mathrm{Cdc} 42$ and the 364 cytoskeleton. FEBS Lett 2003, 542, 7-11.

365 3. Machesky, L.M. Cytokinesis: IQGAPs find a function. Curr Biol 1998, 8, R202-5.

366 4. Mateer, S.C.; Wang, N; Bloom. G.S. IQGAPs: integrators of the cytoskeleton, cell

367 adhesion machinery, and signaling networks. Cell Motil Cytoskeleton 2003, 55, 147-155.

368 5. Weissbach, L.; Settleman, J.; Kalady, M.F.; Snijders, A.J.; Murthy, A.E.; Yan, Y.X.;

369 Bernards, A. Identification of a human rasGAP-related protein containing calmodulin-

370 binding motifs. J Biol Chem 1994, 269, 20517-20521.

371 6. Brill, S.; Li, S.; Lyman, C.W.; Church, D.M.; Wasmuth, J.J.; Weissbach, L.; Bernards, A.;

372 Snijders, A.J. The Ras GTPase-activating-protein-related human protein IQGAP2 harbors a

373 potential actin binding domain and interacts with calmodulin and Rho family GTPases. Mol

374 Cell Biol 1996, 16, 4869-4878.

375

7. Hart, M.J.; Callow, M.G.; Souza, B.; Polakis, P. IQGAP1, a calmodulin-binding protein

376 with a rasGAP-related domain, is a potential effector for cdc42Hs. EMBO J 1996, 15, 2997 -

3773005.

378 8. Osman, M.A.; Cerione, R.A. Iqg1p, a yeast homologue of the mammalian IQGAPs,

379 mediates cdc42p effects on the actin cytoskeleton. J Cell Biol 1998, 142, 443-455.

380 9. Brown, M.D.; Sacks, D.B. IQGAP1 in cellular signaling: bridging the GAP. Trends Cell 381 Biol 2006, 16, 242-249.

382 10. Malarkannan, S.; Awasthi, A.; Rajasekaran, K.; Kumar, P.; Schuldt, K.M.; Bartoszek, A,;

383 Manoharan, N.; Goldner, N.K.; Umhoefer, C.M.; Thakar, M.S. IQGAP1: A Regulator of 384 Intracellular Spacetime Relativity. J Immunol 2012, 188, 2057-2063. 
Kaibuchi, K. Identification of IQGAP as a putative target for the small GTPases, Cdc42 and Rac1. J Biol Chem 1996, 271, 23363-23367.

12. Erickson, J.W.; Cerione, R.A.; Hart, M.J. Identification of an actin cytoskeletal complex that includes IQGAP and the Cdc42 GTPase. J Biol Chem 1997, 272, 24443-24447. 13. Ho, Y.D.; Joyal, J.L.; Li, Z.; Sacks, D.B. IQGAP1 integrates $\mathrm{Ca}^{2+} /$ calmodulin and Cdc42 signaling. J Biol Chem 1999, 274, 464-470. IQGAP1. J Biol Chem 2003, 278, 4347-4352. characterization of the Cdc42-binding site of IQGAP1. Biochem Biophys Res Commun 2003, $305,315-321$.

16. Li, Q.; Stuenkel, E.L. Calcium negatively modulates calmodulin interaction with IQGAP1. Biochem Biophys Res Commun 2004, 317, 787-795. 17. Yamaoka-Tojo, M.; Ushio-Fukai, M.; Hilenski, L.; Dikalov, S.I.; Chen, Y.E.; Tojo, T.; Fukai, T.; Fujimoto, M.; Patrushev, N.A.; Wang, N.; Kontos, C.D.; Bloom, G.S.; Alexander, R.W. IQGAP1, a novel vascular endothelial growth factor receptor binding protein, is involved in reactive oxygen species--dependent endothelial migration and proliferation. Circ Res 2004, 95, 276-283.

404 18. Roy, M.; Li, Z.; Sacks, D.B. IQGAP1 binds ERK2 and modulates its activity. J Biol 405 Chem 2004, 279, 17329-17337.

406 19. Roy, M.; Li, Z.; Sacks, D.B. IQGAP1 is a scaffold for mitogen-activated protein kinase 407 signaling. Mol Cell Biol 2005, 25, 7940-7952.

408 20. Jeong, H.W.; Li, Z.; Brown, M.D.; Sacks, D.B. IQGAP1 binds Rap1 and modulates its 409 activity. J Biol Chem 2007, 282, 20752-20762. 
410 21. Owen, D.; Campbell, L.J.; Littlefield, K.; Evetts, K.A.; Li, Z.; Sacks, D.B.; Lowe, P.N.;

411 Mott, H.R. The IQGAP1-Rac1 and IQGAP1-Cdc42 interactions: interfaces differ between

412 the complexes. J Biol Chem 2008, 283, 1692-1704.

413 22. Jang, D.J.; Ban, B.; Lee, J.A. Characterization of novel calmodulin binding domains

414 within IQ motifs of IQGAP1. Mol Cells 2001, 32, 511-518.

415 23. Atcheson, E.; Hamilton, E.; Pathmanathan, S.; Greer, B.; Harriott, P.; Timson, D.J. IQ-

416 motif selectivity in human IQGAP2 and IQGAP3: binding of calmodulin and myosin

417 essential light chain. Biosci Rep 2011, 31, 371-379.

418 24. Elliott, S.F.; Allen, G.; Timson, D.J. Biochemical analysis of the interactions of IQGAP1

419 C-terminal domain with CDC42. World J Biol Chem 2012, 3, 53-60.

420 25. Fukata, M.; Watanabe, T.; Noritake, J.; Nakagawa, M.; Yamaga, M.; Kuroda, S;

421 Matsuura, Y.; Iwamatsu, A; Perez, F.; Kaibuchi, K. Rac1 and Cdc42 capture microtubules

422 through IQGAP1 and CLIP-170. Cell 2002, 109, 873-885.

423 26. Watanabe, T.; Wang, S.; Noritake, J.; Sato, K.; Fukata, M.; Takefuji, M.; Nakagawa, M.;

424 Izumi, N.; Akiyama, T.; Kaibuchi, K. Interaction with IQGAP1 links APC to Rac1, Cdc42,

425 and actin filaments during cell polarization and migration. Dev Cell 2004, 7, 871-883.

426 27. Morris, L.E.; Bloom, G.S.; Frierson, H.F. ,Jr; Powell, S.M. Nucleotide variants within the

427 IQGAP1 gene in diffuse-type gastric cancers. Genes Chromosomes Cancer 2005, 42, 280-

428286.

429 28. Leung, J.; Yueh, A.; Appah, F.S.,Jr; Yuan, B.; de los Santos, K.; Goff, S.P. Interaction of

430 Moloney murine leukemia virus matrix protein with IQGAP. EMBOJ 2006, 25, 2155-2166.

431 29. Johnson, M.; Sharma, M.; Henderson, B.R. IQGAP1 regulation and roles in cancer. Cell

432 Signal 2009, 21, 1471-1478.

433 30. White, C.D.; Brown, M.D.; Sacks, D.B. IQGAPs in cancer: a family of scaffold proteins

434 underlying tumorigenesis. FEBS Lett 2009, 583, 1817-1824. 
435 31. Sbroggio, M.; Carnevale, D.; Bertero, A.; Cifelli, G.; De Blasio, E.; Mascio, G.; Hirsch, 436 E.; Bahou, W.F.; Turco, E.; Silengo, L.; Brancaccio, M.; Lembo, G.; Tarone, G. IQGAP1 437 regulates ERK1/2 and AKT signalling in the heart and sustains functional remodelling upon 438 pressure overload. Cardiovasc Res 2011, 91, 456-464.

439 32. Sbroggio, M.; Bertero, A.; Velasco, S.; Fusella, F.; De Blasio, E.; Bahou, W.F.; Silengo, 440 L.; Turco, E.; Brancaccio, M.; Tarone, G, ERK1/2 activation in heart is controlled by 441 melusin, focal adhesion kinase and the scaffold protein IQGAP1. J Cell Sci 2011, 124, 3515442 3524.

33. Xie, Y.; Yan, J.; Cutz, J.C.; Rybak, A.P.; He, L.; Wei, F.; Kapoor, A.; Schmidt, V.A.;

444 Tao, L.; Tang, D. IQGAP2, A candidate tumour suppressor of prostate tumorigenesis.

445 Biochim Biophys Acta 2012, 1822, 875-884.

446 34. Wang, C.H.; Balasubramanian, M.K.; Dokland, T. Structure, crystal packing and 447 molecular dynamics of the calponin-homology domain of Schizosaccharomyces pombe Rng2. 448 Acta Crystallogr D Biol Crystallogr 2004, 60, 1396-1403.

449 35. Kurella, V.B.; Richard, J.M.; Parke, C.L.; Lecour, L.F.,Jr; Bellamy, H.D.; Worthylake, 450 D.K. Crystal structure of the GTPase-activating protein-related domain from IQGAP1. J Biol 451 Chem 2009, 284, 14857-14865.

452 36. Umemoto, R.; Nishida, N.; Ogino, S.; Shimada, I. NMR structure of the calponin 453 homology domain of human IQGAP1 and its implications for the actin recognition mode. $J$ 454 Biomol NMR 2010, 48, 59-64.

455 37. Dixon, M.J.; Gray, A.; Schenning, M.; Agacan, M.; Tempel, W.; Tong, Y.; Nedyalkova, 456 L.; Park, H.W.; Leslie, N.R.; van Aalten, D.M.; Downes, C.P.; Batty, I.H. IQGAP proteins 457 reveal an atypical phosphoinositide (aPI) binding domain with a pseudo C2 domain fold. $J$ 458 Biol Chem 2012, 287, 22483-22496. 
38. Shannon, K.B.; Li, R. The multiple roles of Cyk1p in the assembly and function of the

460

461

462

463

464

465

466

467

468

469

470

471

472

473

474

475

476

477

478

479

480

481

482 actomyosin ring in budding yeast. Mol Biol Cell 1999, 10, 283-296.

39. Mateer, S.C.; Morris, L.E.; Cromer, D.A.; Bensenor, L.B.; Bloom, G.S. Actin filament binding by a monomeric IQGAP1 fragment with a single calponin homology domain. Cell Motil Cytoskeleton 2004, 58, 231-241.

40. Mateer, S.C.; McDaniel, A.E.; Nicolas, V.; Habermacher, G.M.; Lin, M.J.; Cromer, D.A.; King, M.E.; Bloom, G.S. The mechanism for regulation of the F-actin binding activity of IQGAP1 by calcium/calmodulin. J Biol Chem 2002, 277, 12324-12333.

41. Bashour, A.M.; Fullerton, A.T.; Hart, M.J.; Bloom, G.S. IQGAP1, a Rac- and Cdc42binding protein, directly binds and cross-links microfilaments. J Cell Biol 1997, 137, 15551566.

42. Pelikan-Conchaudron; A.; Le Clainche; C.; Didry, D.; Carlier, M.F. IQGAP1 is a calmodulin-regulated barbed end capper of actin filaments: possible implications in its function in cell migration. J Biol Chem 2011, 286, 35119-35128.

43. Pathmanathan, S.; Hamilton, E.; Atcheson, E.; Timson, D.J. The interaction of IQGAPs with calmodulin-like proteins. Biochem Soc Trans 2011, 39, 694-699.

44. Andrews, W.J.; Bradley, C.A.; Hamilton, E.; Daly, C.; Mallon, T.; Timson, D.J. A calcium-dependent interaction between calmodulin and the calponin homology domain of human IQGAP1. Mol Cell Biochem 2012, 371, 217-223.

45. Bradford, M.M. A rapid and sensitive method for the quantitation of microgram quantities of protein utilizing the principle of protein-dye binding. Anal Biochem 1976, 72, $248-254$.

46. Kelley, L.A.; Sternberg, M.J. Protein structure prediction on the Web: a case study using the Phyre server. Nat Protoc 2009, 4, 363-371. 
47. Krieger, E.; Joo, K.; Lee, J.; Lee, J.; Raman, S.; Thompson, J.; Tyka, M.; Baker, D.;

484 Karplus, K. Improving physical realism, stereochemistry, and side-chain accuracy in homology modeling: Four approaches that performed well in CASP8. Proteins 200977 Suppl 9, 114-122.

48. Duhovny, D.; Nussinov, R.; Wolfson, H. Efficient unbound docking of rigid molecules. Algorithms in Bioinformatics, Proceedings 2002, 2452, 185-200.

50. Farrell, D.W.; Speranskiy, K.; Thorpe, M.F. Generating stereochemically acceptable

493 protein pathways. Proteins 2010, 78, 2908-2921.

494 51. de Vries, S.J.; van Dijk, M.; Bonvin, A.M. The HADDOCK web server for data-driven biomolecular docking. Nat Protoc 2010, 5, 883-897.

498 53. Kahraman, A.; Malmstrom, L.; Aebersold, R. Xwalk: computing and visualizing 499 distances in cross-linking experiments. Bioinformatics 2011, 27, 2163-2164.

500 54. Costantini, S.; Colonna, G.; Facchiano, A.M. ESBRI: a web server for evaluating salt 501 bridges in proteins. Bioinformation 2008, 3, 137-138.

502 55. Tina, K.G.; Bhadra, R.; Srinivasan, N. PIC: Protein Interactions Calculator. Nucleic Acids 503 Res 2007, 35, W473-W476.

504 56. Fraczkiewicz, R.; Braun, W. Exact and efficient analytical calculation of the accessible surface areas and their gradients for macromolecules. J Comput Chem 1998, 19, 319-333. 

new bioinformatics analysis tools framework at EMBL-EBI. Nucleic Acids Res 2010, 38, W695-W699.

58. Sievers, F.; Wilm, A.; Dineen, D.; Gibson, T.J.; Karplus. K.; Li, W.; Lopez, R.;

510 McWilliam, H.; Remmert, M.; Soding, J.; Thompson, J.D.; Higgins, D.G. Fast, scalable generation of high-quality protein multiple sequence alignments using Clustal Omega. Mol Syst Biol 2011, 7, 539.

59. Altschul, S.F.; Madden, T.L.; Schaffer, A.A.; Zhang, J.; Zhang, Z.; Miller, W.; Lipman, D.J. Gapped BLAST and PSI-BLAST: a new generation of protein database search programs.

$515 \quad$ Nucleic Acids Res 1997, 25, 3389-3402.

516 60. Valdar, W.S. Scoring residue conservation. Proteins 2002, 48, 227-241.

517 61. Linding, R.; Jensen, L.J.; Diella, F.; Bork, P.; Gibson, T.J.; Russell, R.B. Protein disorder 518 prediction: implications for structural proteomics. Structure 2003, 11, 1453-1459.

519 62. McGuffin, L.J. Intrinsic disorder prediction from the analysis of multiple protein fold 520 recognition models. Bioinformatics 2008, 24, 1798-1804.

521 63. Saha, R.P.; Bahadur, R.P.; Pal, A.; Mandal, S.; Chakrabarti, P. ProFace: a server for the 522 analysis of the physicochemical features of protein-protein interfaces. BMC Struct Biol 2006, $6,11$.

64. Tegge, A.N.; Wang, Z.; Eickholt, J.; Cheng, J. NNcon: improved protein contact map prediction using 2D-recursive neural networks. Nucleic Acids Res 2009, 37, W515-W518. 65. Cheng, J.; Randall, A.; Baldi, P. Prediction of protein stability changes for single-site mutations using support vector machines. Proteins 2006, 62, 1125-1132. 
531 67. Topham, C.M.; Srinivasan, N.; Blundell, T.L. Prediction of the stability of protein mutants based on structural environment-dependent amino acid substitution and propensity tables. Protein Eng 1997, 10, 7-21.

68. Dyson, H.J.; Wright, P.E. Coupling of folding and binding for unstructured proteins. Curr Opin Struct Biol 2002, 12, 54-60.

69. Dyson, H.J.; Wright, P.E. Intrinsically unstructured proteins and their functions. Nat Rev Mol Cell Biol 2005, 6, 197-208.

70. Tompa, P. The interplay between structure and function in intrinsically unstructured proteins. FEBS Lett 2005, 579, 3346-3354. actin:DNase I complex. Nature 1990, 347, 37-44. Laboratory Press, Cold Spring arbor, New York.

73. Adachi, H.; Takahashi, Y.; Hasebe, T.; Shirouzu, M.; Yokoyama, S.; Sutoh, K.

Dictyostelium IQGAP-related protein specifically involved in the completion of cytokinesis. J

546 Cell Biol 1997, 137, 891-898.

74. Sakurai, M.; Adachi, H.; Sutoh, K. Mutational analyses of Dictyostelium IQGAP-related protein GApa: possible interaction with small GTPases in cytokinesis. Biosci Biotechnol Biochem 2001, 65, 1912-1916. 75. Levine, B.A.; Moir, A.J.; Patchell, V.B.; Perry, S.V. The interaction of actin with dystrophin. FEBS Lett 1990, 263, 159-162. 76. Levine, B.A.; Moir, A.J.; Patchell, V.B.; Perry, S.V. Binding sites involved in the interaction of actin with the N-terminal region of dystrophin. FEBS Lett 1992, 298, 44-48. 77. Kuhlman, P.A.; Hemmings, L.; Critchley, D.R. The identification and characterisation of an actin-binding site in $\alpha$-actinin by mutagenesis. FEBS Lett 1992, 304, 201-206. 
556 78. Gimona, M.; Mital, R. The single $\mathrm{CH}$ domain of calponin is neither sufficient nor 557 necessary for F-actin binding. J Cell Sci 1998, 111 ( Pt 13), 1813-1821.

558 
560 Figure 1: Crosslinking of the CHD of human IQGAP1 (CHD1, $27 \mu \mathrm{M})$ and actin $(23 \mu \mathrm{M})$ by

561 EDC. The products of the reaction were resolved by $12.5 \%$ SDS-PAGE and visualised by

562 staining with Coomassie blue. M, molecular mass markers (masses are shown to the left of

563 the gel in $\mathrm{kDa})$; A, actin; C, CHD1; AC actin and CHD. In the control lanes, CHD1 (27 $\mu \mathrm{M})$

564 and actin $(23 \mu \mathrm{M})$ were incubated with crosslinker under identical conditions. The additional

565 bands in the actin lane most likely represent actin dimers and oligomers. Note that, due to the

566 equilibrium nature of the interaction and the short half-life of EDC, the crosslinking reaction

567 does not go to completion. Therefore, some non-crosslinked product remains visible in lane

568 AC.

$570 \quad$ Figure 2: Predicted structure of the CHD1/ $\alpha$-actin complex. $\alpha$-Actin is shown on the left of both space-filling (top) and cartoon (bottom left) models in green. An enlarged image of the potential interaction site and key residues is also shown (bottom right). $\alpha$-Actin residue labels are in green whilst those belonging to CHD1 are in blue.

Figure 3: FIRST/FRODAN flexibility predictions for (a) CHD1 and (b) $\alpha$-actin. DISOclust predicted disordered regions in (c) CHD1 and (d) $\alpha$-actin

Figure 4: Alignments of the predicted structures of the CHD from the IQGAP-like proteins from (a) C. elegans, (b) H. vulgaris and (c) S. cerevisiae with human CHD1 (green in all three alignments). (d) D. discoideum IQGAP-like protein gapA has an atypical CHD-like

581 structure at the N-terminus. Structural alignment of this domain with human CHD1 showing coincident polar, charged residues (human CHD1 in green). 
584 Supplementary Figure S1: Results from the Proface server revealed surface-exposed,

585 potentially interacting residues.

586

587 Supplementary Figure S2: Results from DisEMBL reveal potentially flexible residues

588 (highlighted in yellow).

589

590 Supplementary Figure S3: Protein sequence alignments of the N-termini of IQGAP-like 591 proteins from a variety of species.

592

593 